\title{
Improving the efficiency of routing solution in elastic optical networks
}

\author{
Majid Forghani-elahabad ${ }^{1}$, Luiz H. Bonani \\ Center for Engineering, Modeling and Applied Social Sciences (CECS), UFABC, \\ Santo André, SP, Brazil
}

\begin{abstract}
Transparent elastic optical networks (EONs) have been proposed as a promising solution to improve the efficiency of spectrum utilization for the ever-increasing Internet traffic demand. Routing and spectrum assignment (RSA) problem is a very attractive problem in EONs, and several computational algorithms have been proposed for the problem treating it as a single problem or as two sub-problems: routing and spectrum assignment. Here, we consider the routing sub-problem in a dynamic case and propose two strategies to improve the solution. In the first one, assuming that the first $k$ shortest paths (SPs) are predetermined for each node pair, we define a new cost for each SP, and rearrange the SPs with respect to the new costs. Then, we firstly check the SP with less cost for assigning spectrum. This way, the blocking probability is expected to decrease. In the second strategy, a predetermined partitioning of the frequency slots (FSs) on each link is considered, the number of free blocks on each link is updated after each spectrum assignment, and upon arriving a new connection request, we find the SP considering links' length as the number of used blocks of the requested bandwidth size. This way, decreasing the wasting of spectrum on the SPs, the blocking probability is expected to decrease as well.
\end{abstract}

Keywords: Elastic optical networks, Routing and spectrum assignment, Blocking probability.

\section{Introduction}

In recent years, Internet traffic demand has been doubling nearly every two years and it is predicted that this growth continues in the next years due to ever-increasing demand for Internet services such as high-definition and Video on Demand [1]. Hitherto, wavelength division multiplexing (WDM) technology has been extensively employed in backbone optical networks due

\footnotetext{
${ }^{1}$ E-mail Corresponding Author: m.forghani@ufabc.edu.br
} 
to its capability to transmit huge amount of traffic concurrently by dividing the bandwidth of a fiber into multiple channels [2]. However, a notable point about WDM technology is its rigid and coarse granularity leading to allocation of a whole wavelength to a connection even if it is not necessary. This results in low scalability, high cost, and inefficient capacity utilization. Hence, there have been a need for a more efficient, flexible, and scalable direction network design and infrastructure. To address this necessity, some technologies such as optical burst switching, optical virtual concatenation, and optical packet switching have been worked out, however, due to the immaturity, they have not been considered as the solution [3]. Nowadays, orthogonal frequency-division multiplexing (OFDM) has been considered a very promising solution for optical transmission and networking. OFDM technology splits a data stream into multiple consecutive subcarriers (frequency slots (FSs)) in order to increase the flexibility leading to the elastic optical network (EON) architectures [4]. Considering EONs, the optical spectrum can be used in a more flexible way with a finer granularity in order to achieve high scalability, low cost, and efficient capacity utilization. As a result, EONs have been considering the upgraded version of WDM optical networks [4]-[12].

One of the most attractive problems in WDM networks is routing and wavelength assignment (RWA) problem which has been extensively worked out in the last decades $[13,14]$. The equivalent problem in EONs has been introduced as routing and spectrum assignment (RSA) which is more complicated than RWA problem since the number of FSs is exponentially increased [2]-[12]. In fact, the efficacy of EONs is really related to the employed technique for solving the RSA problem. According to the connection requests' types, static and dynamic RSA problems have been studied in the literature [5]-[12]. In the static case, the whole list of connection requests are known in advance, and usually the aim is to set up lightpaths such that the utilized resources are minimized, or the number of appointed connections is maximized by a fixed amount of resources while the established lightpaths will remain indefinitely in the network $[6,7]$. The proposed algorithms for static RSA problem have usually formulated it as an integer linear programming [6], and also there have been proposed some heuristic techniques to address it [7]. In the dynamic case, the connection requests arrive in dynamical times, and hence a lightpath is set up upon arriving a connection request and will be released after accomplishing the request, but this time is not known a priori [9]-[12]. There are two important constraints to be considered for both static and dynamic RSA problems: spectrum continuity that says the same subcarriers should be assigned on all the links of a path, and 
spectrum contiguity that says the devoted subcarriers to a connection should be consecutive. There are some other constraints such as guard bands that says the adjacent connections should be separated using at least one undedicated subcarrier. We also note that on a same link at any time, a subcarrier can be used only for one connection. The RSA problem is NP-complete [8]. Several different metrics such as (call or bandwidth) blocking probability, shareability, spectrum utilization, energy efficiency, and fragmentation have been used to evaluate the algorithms on RSA problem. Moreover, some authors have studied the fairness or unfairness rates of the algorithms [9]-[11].

The RSA problem can be considered as a single problem or divided into two sub-problems: the routing one and the spectrum assignment one. Here, we consider the routing sub-problem. For this problem, usually, the algorithms considered some primary and backup routes which are often separated. Adaptive routing and fixed-alternate routing are two known techniques for routing sub-problem. In the first one, we determine the best adaptive path after arriving a new connection request, and in the later one, given the first $k$ shortest paths (SPs), we check the SPs one by one to find enough resources for assigning spectrum. We propose two strategies to improve these two techniques. A strategy to improve the fixed-alternate routing technique and another one for proposing an improved adaptive routing method. Next, the strategies are proposed in Section 2. Section 3 provides some numerical results, and the conclusion remarks are given in Section 4 .

\section{Two strategies}

Let $G(N, E, F)$ be an elastic optical network (EON) with $N=\{1,2$, $\cdots, n\}$ being the set of nodes, $E=\left\{e_{1}, e_{2}, \cdots, e_{m}\right\}$ being the set of edges (links), and $F$ being the total number of available frequency slots (FS) on each fiber link. We consider the RSA problem as two sub-problems: (1) routing problem, and (2) spectrum assignment problem. There are several techniques for spectrum assignment such as first fit, random fit, min void, and max void [10]. Some algorithms have divided the available spectrum to different partitions in order to reduce fragmentation [12]. However, our focus is on routing sub-problem proposing two strategies. We note that in both strategies a partitioning scenario is used for spectrum assignment. Assuming $\left\{d_{1}, d_{2}, \cdots, d_{q}\right\}$ as the set of all the demand sizes with a uniform distribution function, we make a predetermined partitioning for the frequency slots (FSs) on each link. This partitioning considers almost the same number of blocks for each demand size. For example, if there are 60 FSs on each link with five demand sizes $\{2,4,6,8,10\}$, we consider two blocks for each demand size as 
it is seen in Fig. 1.

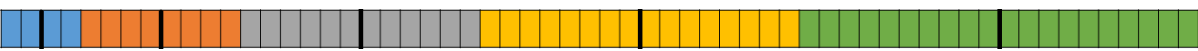

Figure 1 - A sample of partitioning a link with $60 \mathrm{FSs}$ and five demand sizes $\{2,4,6,8,10\}$.

\subsection{The first Strategy}

In this strategy, we assume that the first $k$ shortest paths (SPs) for each node pair are determined in advance, and we rearrange the order of the SPs by using a new defined cost in order to decrease the call blocking probability. Assume that $N P=\{(i, j) \mid i$ is a source and $j$ is a sink $\}$ is the set of all the node pairs in the network. For each node pair $(i, j) \in N P$, $K_{i j}=\left\{P_{i j}^{1}, P_{i j}^{2}, \cdots, P_{i j}^{k}\right\}$ denotes the first $k$ predetermined SPs from $i$ to $j$. Moreover, $K=\cup_{(i, j) \in N P} K_{i j}=\left\{P_{1}, P_{2}, \cdots, P_{\kappa}\right\}$ denotes the set of all the predetermined SPs in the network, considering all the node pairs, in which $\kappa=|K|$ is the number of all the SPs. Let $\delta_{s r}=(0) 1$ when SP, $P_{s}$ (does not) comprise link $e_{r}$, for $s=1,2, \cdots, \kappa$, and $r=1,2, \cdots, m$. Now, for each link in an EON, say $e_{r}$, a cost of $c_{r}$ is defined as follows.

$$
c_{r}=\sum_{s=1}^{\kappa} \delta_{s r}, \quad \text { for } r=1,2, \cdots, m .
$$

The cost of each SP is considered as sum of its entire links' costs given in the following equation:

$$
C_{s}=\sum_{e_{r}: e_{r} \in P_{s}} c_{r}, \quad \text { for each } s=1,2, \cdots, \kappa .
$$

We note that $c_{r}$ denotes the cost of link $e_{r}$ and $C_{s}$ denotes the cost of SP, $P_{s}$. After computing the costs, one can rearrange the $k$ predetermined SPs for each node pair according to the newly obtained ascending costs. We note that the costs are computed once, before arriving any connection requests, and hence this computational process does not affect on the complexity results of the routing approach. The practical efficiency of the strategy is demonstrated in Section 3.

\subsection{The second Strategy}

Here, we propose an adaptive routing method for dynamic RSA problem using the predetermined partitioning explained through Fig. 1. This 
partitioning is considered in [11] to propose a fairness behavior. However, we consider it to define a new length on the links in the network. In fact, assuming $\left\{d_{1}, d_{2}, \cdots, d_{q}\right\}$ as the set of all the demand sizes, for each link, say $e_{r}$, we consider a length vector $\left(l_{1}^{r}, l_{2}^{r}, \cdots, l_{q}^{r}\right)$, for $r=1,2, \cdots, m$, in which $l_{i}^{r}$ is the number of occupied blocks of size $d_{i}$ on link $e_{r}$. Then, upon arriving a connection request of a size, say $d_{j}$, we determine the SP considering the $j$ th components of length vectors. This way, we determine a path with the minimum number of occupied blocks of size $d_{i}$. Moreover, to be sure that all the links of the determined SP have at least one free block of the related demand size, we set a length component, say $l_{i}^{r}$, to infinity when all the blocks of size $d_{i}$ are occupied on link $e_{r}$. It is vivid that if there is no path with finite length, then the connection request should be blocked in this strategy. Since we need to update the network information after each lightpath establishment, the complexity of this strategy is somewhat high, however the call blocking probability is decreased along with increasing the fairness of the algorithm. The numerical results in the next section show the efficiency of this strategy.

\section{Numerical results}

Here, we make some numerical results to show the efficiency of the proposed strategies. The numerical results are provided using the Manhattan topology given in Fig. 2. As our focus is on routing sub-problem, for more convenient, we consider a predetermined partitioning for the FSs on each link (explained in Section 2.2), and to assign spectrum to a connection request, we utilize the available blocks on the associated partition with the demand size if it is possible, otherwise the connection is blocked. It is also assumed that the demand sizes are $\{2,4,6,8,10\}$ with the same probability, i.e., uniform distribution function, and each fiber link contains 300 FSs. Hence, we partition each link to five parts which one contains ten blocks of an associated size. The arrival times of every connection request follow a Poisson distribution with an average of $\lambda$ connection requests per time unit. The service times follow an exponential distribution with average service time $1 / \mu$ equal to 10 time units. We consider all the possible node pairs on the network $(380=20 \times 19$ node pairs $)$, and the connection requests select a given node pair following a uniform distribution. Moreover, the guard bands are not considered. We generated $5 \times 10^{5}$ connection requests. In addition, to guarantee the same input conditions for both techniques, a random number generator with a constant seed is considered. 


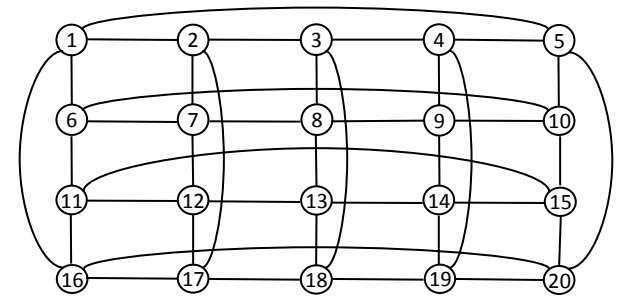

Figure 2 - Bidirectional Manhattan topology with 20 nodes.

In the first strategy, a fixed-alternative routing technique is proposed. Given the first $k$ SPs for each node pair, we propose a new arrangement of the SPs to decrease the call blocking probability. Hence, to show the practical efficacy of the strategy, we compare Algorithm 1 (based on our strategy) that checks the rearranged SPs to assign spectrum with Algorithm 2 (a basic one) that checks the SPs by their initial order to assign spectrum. The number of SPs for each node pair is set as $k=3$. Fig. 3 depicts the final results demonstrating the practical efficiency of our strategy.

In the second strategy, we propose an adaptive routing technique. To show its practical efficiency, we compare Algorithm 3 (based on our strategy) that finds the SP according to the newly defined lengths and checks it for assigning spectrum with Algorithm 3 (a basic one) that determines the SP according to the number of hops and checks it for assigning spectrum. We block the connection in both algorithms when there is not available resources in the first selected SP. Fig. 4 depicts the final results showing the significant practical efficiency of our strategy especially for the low loads.

Moreover, Fig. 5 shows the numerical results for comparing all the four algorithms. As it is seen, for low loads, Algorithm 3 gives the best results even though it uses only one SP to check for assigning spectrum. However, for high loads, Algorithm 1 gives the best results. 


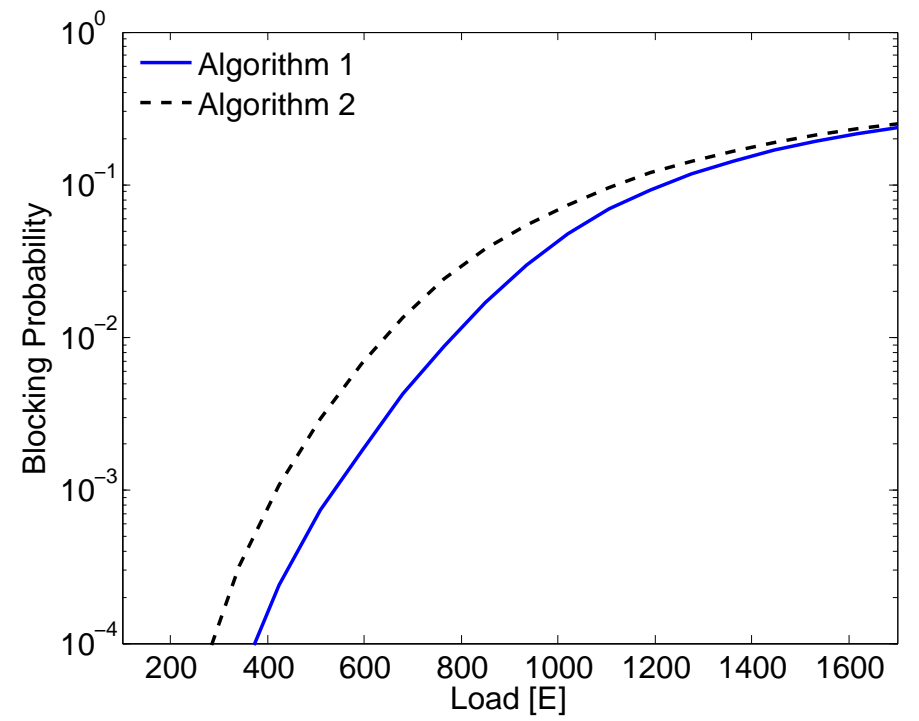

Figure 3 - The final result for comparing algorithms 1 and 2 to show the efficiency of the first strategy.

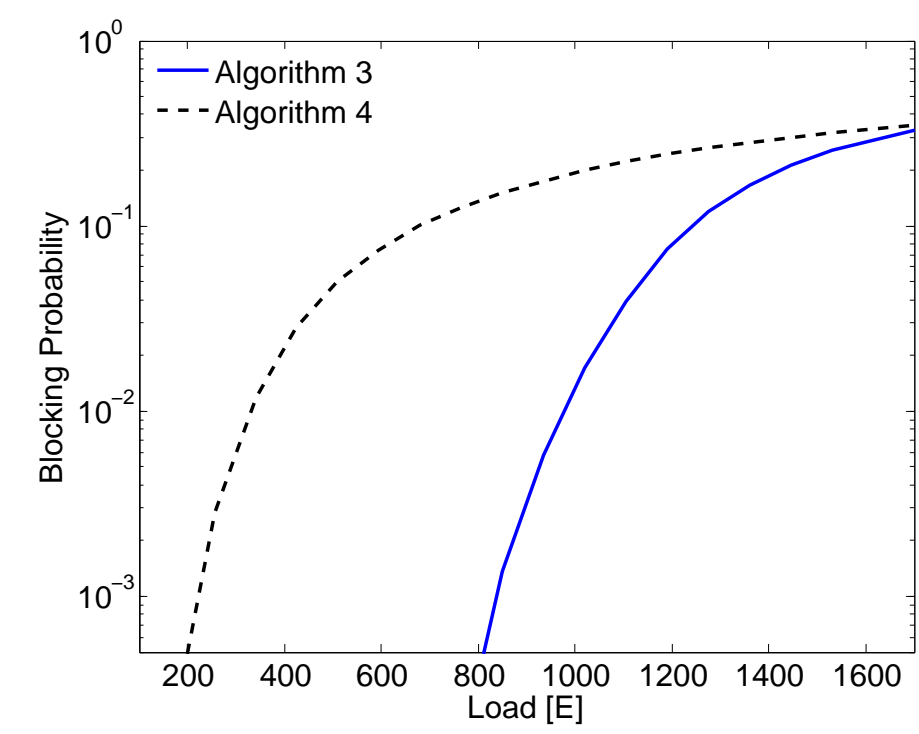

Figure 4 - The final result for comparing techniques 3 and 4 to show the efficiency of the second strategy. 


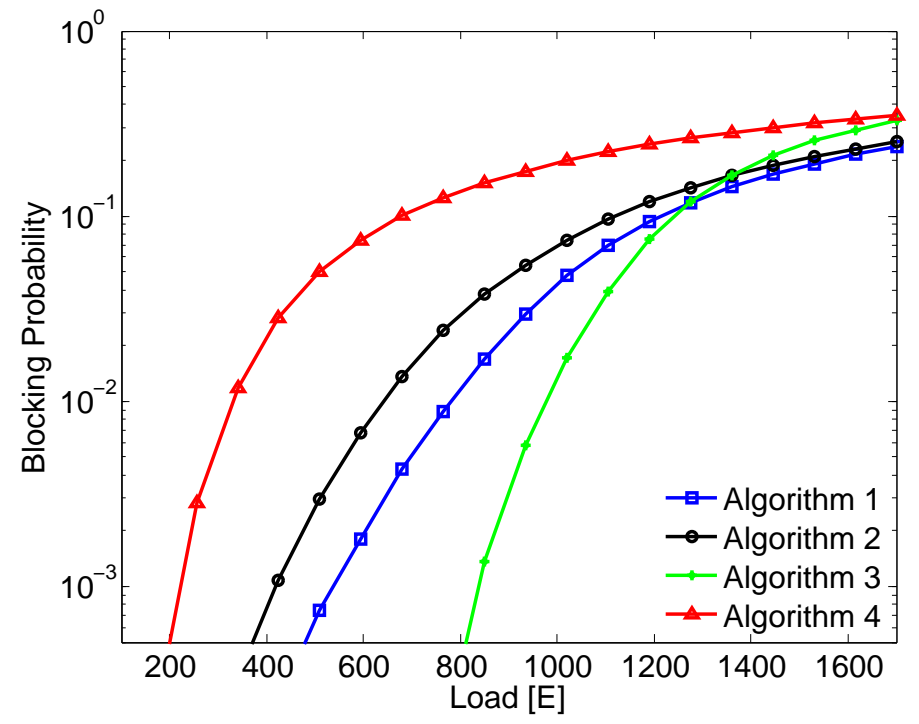

Figure 5 - The final result for comparing techniques 3 and 4 to show the efficiency of the second strategy.

\section{Conclusions}

Routing and spectrum assignment (RSA) problem is one of the most attractive problems in elastic optical networks (EONs). Several algorithms have been proposed considering either static or dynamic cases of the problem. Some works have treated the problem as a single problem, and some others have decoupled it into two sub-problems of routing one and spectrum assignment one. Here, we considered the routing sub-problem, and proposed two strategies to improve the solution. The first strategy improved the fixed-alternate routing technique, and the second one proposed an improved adaptive routing method. We made some numerical experiments using a bidirectional Manhattan topology with 20 nodes. The numerical results demonstrated the practical efficacy of our proposed strategies (Algorithm 1 and Algorithm 3) in comparison with the basic ones (Algorithm 2 and Algorithm 4). It was also shown that for the high loads, the first strategy is the best, and for the low loads, the second strategy is the best.

Acknowledgments. The first author thanks the PNPD/CAPES (grant 23038.009634/2016-71) and the second author thanks CNPq (grant 306153/20130 ) for supporting this work. 


\section{References}

[1] Saleh, A.A.M, Simmons, J.M. Technology and Architecture to Enable the Explosive Growth of the Internet, IEEE Communications Magazine 49(1): 126-132, 2011.

[2] Bandyopadhyay, S. Dissemination of Information in Optical Networks, 2008, Springer Berl, Heidelb.

[3] Tucker, R.S. Optical packet switching: A reality check, Optical Switching and Networking 5(1): 2-9, 2008.

[4] Zhang, G., Leenheer, M.D., Morea, A., Mukherjee, B. A survey on OFDM-based elastic core optical networking, IEEE Communications Surveys Tutorials 15(1): 65-87, 2013.

[5] Shieh W., Djordjevic, I. OFDM for Optical Communications, 2009, Elsevier Science.

[6] Klinkowski, M., Walkowiak K. Routing and Spectrum Assignment in Spectrum Sliced Optical Path Network, IEEE Communications Letters 15(8): 884-886, 2011.

[7] Vizcaino, J.L., Ye,Y., Monroy, I.T. Energy efficiency analysis for flexible-grid OFDM-based optical networks, Computer Networks 56(10): 2400-2419, 2012.

[8] Christodoulopoulos, K., Tomkos, I., Varvarigos, E.A. Elastic Bandwidth Allocation in Flexible OFDM-Based Optical Networks, Journal of Lightwave Technology 29(9): 1354-1366, 2011.

[9] Callegati, F., Bonani, L.H., Cerroni, W. Service fairness in flexible optical networks, in Proc. 2013 OFC, paper no. OTh1H.4.

[10] Callegati, F., Bonani, L.H., Lezama, F., Cerroni, W., Campi, A, Castanon, G. Trunk reservation for fair utilization in flexible optical networks, IEEE Communication Letters 18(5): 889-892, 2014.

[11] Rodrigo Tessinari, R., Puype, B., Colle, D., Garcia, A. Zone Based Spectrum Assignment in Elastic Optical Networks: A Fairness Approach, Opto-Electronics and Communications Conference (OECC), Shanghai, China, 2015. 
[12] Gerstel, O., Jinno, M., Lord, A. Elastic optical networking: a new dawn for the optical layer?, IEEE Communications Magazine 50: s12-s20, 2012.

[13] Zang, H., Jue, J.P. A review of routing and wavelength assignment approaches for wavelength-routed optical WDM networks, Optical Networks Magazine 1: 47-60, 2000.

[14] Bonani, L.H., Forghani-elahabad, M. An improved least cost routing approach for WDM optical network without wavelength converters, Optical Fiber Technology 32: 30-35, 2016. 